\title{
Squarks and strings more real
}

\author{
Particle physics is again in turmoil. Experimentalists believe they may have new phenomena to describe. \\ Theorists may already have an accommodating theory.
}

THESE are heady days for particle physics. Hardly a week after Dr Carlo Rubbia of CERN had been in Stockholm to collect his Nobel prize for discovering the $\mathrm{W}^{ \pm}$and $\mathrm{Z}^{0}$ particles, which mediate the weak nuclear force, he paid a flying visit to a meeting at the Rutherford Laboratory near Oxford to describe preliminary evidence that the same UA1 experiment may have revealed a counterpart of ordinary matter called "supermatter", in which "matter" (such as electrons) and "radiation" (such as photons) exchange roles.

Many theorists would dearly like to believe Rubbia's evidence. For example, John Ellis, also from CERN (the European Organization for Nuclear Research near Geneva), where Rubbia's UA1 experiment is taking new data on high energy protonantiproton collisions, has risked a bottle of wine in a wager with US theorist Sydney Drell that supermatter will be discovered by next July. Rubbia himself was last week more cautious. Other mysterious events at the CERN collider have sunk under the flood of new data taken in the past three months, he explained. But certain "monojet" (now "bijet") events, with large amounts of missing energy, persist, cannot be explained as experimental artefacts but can be interpreted as evidence for the creation and decay of superparticles. The UAl detectors around the proton antiproton collider at CERN have just come to the end of a run, but it is clear from what Rubbia said two weeks ago that there is still a great deal to be quarried from the data already collected.

Rubbia hopes to be able to make a more confident statement on these events early in the year. Whether his group has truly found supermatter or not, what has the theoreticians agog is that it is already predicted by theories based on the idea of "supersymmetry", which endows every fundamental entity with a counterpart with contrasting quantum statistics. Familiarly, electrons with Fermi statistics obey Pauli's exclusion principle, making chemistry possible. Photons, on the other hand, have Bose statistics, which allows many photons to exist in the same state, and makes intense light beams and electromagnetic fields possible. But superelectrons (selectrons) would have Bose statistics, while superphotons (photinos) would be fermions. Quarks (fermions) would be partnered by squarks (bosons), gluons (bosons) by gluinos (fermions), and so on.

Since supermatter seems not to exist at low energy, theorists have hitherto assumed that the supermatter counterparts of ordinary particles have large masses. But theory has been able to put only vague limits on the masses of superparticles. One interpretation of Rubbia's events, compatible with these limits, is that he is creating $40-\mathrm{GeV}$ squarks, paired with 40-GeV antisquarks, each of which decays into an ordinary quark (or antiquark) and a photino. The photinos interact only weakly with ordinary matter, and carry off the missing energy which is now thought to characterize the special UAl events. The quark and antiquark would each make "jets" of hadronic matter.

Ellis had already predicted that, if squarks have a sufficiently low mass to be produced at UA1, the detector should see pairs of jets plus missing energy. His difficulty had been that Rubbia's early data had shown only single jets. Now, however, Rubbia's group is finding bijets - news that delighted Ellis, who heard it for the first time at the Rutherford meeting. Moreover, the UA1 group can now interpret the early detection of monojets as an artificial experimental bias against bijets. For example, many of the early "monojets" can be seen, on closer inspection, to have had other jets associated with them which were eliminated by a computer code deliberately designed to ignore jets below $12 \mathrm{GeV}$ total energy.

As if the potential discovery of supermatter were not enough, Michael Green of Queen Mary College, London, introduced at the meeting a theory he has developed with John Schwartz of the California Institute of Technology that describes particles as massless relativistic "superstrings". Superstrings are entities in ten dimensions (nine space-like, one time-like) which are expected to behave like ordinary particles when the ten dimensions are collapsed to four, but whose characteristic properties derive from the six hidden dimensions.

For the past several years, theorists have been toying - and more than that - with particle theories written in more than four dimensions. One fashion, for example, has been a sequence of theories in eleven dimensions, in which the extra seven dimensions are supposed at the outset to be closed on themselves with a certain characteristic scale. The properties of particles in the real world can then be recovered as the bubble of non-existent space shrinks to nothing, like the smile on the face of a Cheshire cat. The advantage is that manipulation in many dimensions may be easier than in a mere four.

The theory has already chalked up conspicuous successes, notably the accolade from Professor Edward Witten of Princeton that the theory is "stunning". One of its results is a simple relation between the gravitational constant and the gauge coupling constant (the "charge" which describes the fundamental strength of electromagnetic, strong and weak forces) of the form

$$
x=\text { constant } . g^{2} \cdot T
$$

where $x$ is the Newtonian gravitational constant, $g$ the gauge coupling and $T$ (the tension in the fundamental string) is the only adjustable constant in the theory. This link is related to the simple fact that in this theory nongravitational forces are caused by the exchange of open-ended strings, and gravitational forces by the exchanges of closed loops. The existence of one kind of force implies the existence of the other.

Superstring theory is supersymmetric (so there would be no conflict with the discovery of supermatter), but the real reasons it has caught attention are its links with gravity, its apparent consistency with quantum mechanics and its apparent nearuniqueness.

Green and Schwarz's superstrings in a ten-dimensional space-time have the further advantage that they are inherently chiral, which means that they can describe parity-breaking, an essential for any theory meant to accommodate the weak interaction, as a truly unified theory of the forces must.

Here lies one of the "miracles" of the theory, as Green describes them. Existing grand unified theories are plagued by "anomalies" in charge conservation when they are quantized; the theories are constructed to conserve charge, but when certain simple interactions of particles are calculated, it turns out that charge conservation is violated, in most chiral versions of the theories, unless certain unnatural coincidences are assumed to occur. But in Green and Schwarz's superstring theory, the anomalies cancel each other naturally; moreover, this cancellation involves the exchange of loops, which is another way of saying that gravity is intimately involved. The hint, therefore, is that the long-sought quantum theory of gravity may also be hidden in the theory. It is no wonder that strings are suddenly so fashionable.

Robert Walgate 